\title{
Pneumococcal Meningitis: Current Pathophysiologic Concepts
}

\author{
MERLE A. SANDE ${ }^{1}$ and MARTIN G. TÄUBER ${ }^{2}$
}

$\mathbf{M}^{\mathrm{s}}$ ENINGITIS CAUSED BY Streptococcus pneumoniae has become the most common bacterial infection of the central nervous system. ${ }^{31}$ It is rapidly fatal if not treated early and effectively with antibiotics that achieve bactericidal activity at the site of infection, which is in the subarachnoid space filled with cerebrospinal fluid (CSF). ${ }^{28}$ For the last $40-50$ years, the $\beta$-lactam antibiotics have fulfilled this function. However, with the emergence of resistance to these and other antibiotics, the future approach to treatment of this infection could be problematic. This renewed interest in pneumococcal disease stimulates us to review what we have learned about the pathophysiology of this disease since the development of the Dacey/Sande meningitis model in the early 1970 s. ${ }^{5}$ It has always been our hope that an in-depth understanding of the mediators that lead to abnormalities in brain function and eventual neuronal death would lead to therapeutic interventions that would reduce these destructive consequences. Today, these goals are more important than ever.

\section{THE MODEL}

The model was first described by Dacey and Sande in 1974, when it was used to demonstrate that probenecid increased the concentration of penicillin in the CSF fluid by blocking the organic acid exit pump located in the choroid plexus. ${ }^{5}$ The model consisted of a rabbit suspended in a stereotactic frame that allowed instillation of microorganisms (usually the pneumococcus) directly into the cisterna magna of the animal and then, with the needle left in place, continuous sampling of CSF as the infection developed. CSF could be analyzed for viable bacteria, various mediators and indicators of inflammation, drug concentrations as a function of simultaneous serum concentrations, and CSF pressure. Brain histology and brain water content could be measured at the conclusion of the experiment. ${ }^{36}$ The unique ability to monitor the infection over time allowed for a real-time assessment of the importance of the various components of the inflammatory process and allowed a direct measure of their contribution to the pathophysiology of the disease by the use of selective inhibitors. While the experimental work has expanded into various other in vivo and in vitro models over the past 30 years by numerous investigators, most of the initial observations were made using this rabbit model.

\section{THE INFECTION}

It was found that a very low inoculum of encapsulated pneumococci could produce a progressive infection in the rabbit, ${ }^{27}$ an observation confirmed by Moxon in the infant rat model of Haemophilus influenza type b meningitis, who found that it only took a single organism in the CSF to produce meningitis. ${ }^{21} \mathrm{Un}$ encapsulated mutants were nonpathogenic. Each bacterial strain had its own unique infectious dose $50\left(\mathrm{ID}_{50}\right)$, which is the number of organisms necessary to produce progressive infection in $50 \%$ of the animals when directly injected into the CSF. This proved to be a measure of virulence for the meningitis potential of the various bacteria. With a well-characterized type $3 S$. pneumoniae, the $\mathrm{ID}_{50}$ was less than 100 organisms. After injection, a lag phase with stationary growth lasted for approximately $2 \mathrm{hr}$ and then the bacteria grew with a generation (or doubling) time of approximately $60 \mathrm{~min}$ (compared to $20 \mathrm{~min}$ in broth) until they reached "maximum population densities" of $10^{6}-10^{8} \mathrm{cfu} / \mathrm{ml}$ after $18-36 \mathrm{~h}$. Adapting the model to the dog, we found that shortly after initiation of log-phase growth in CSF, the pneumococcus appeared in the sagittal sinus, followed $2 \mathrm{hr}$ later by appearance in the arterial circulation, thus demonstrating a unique clearance mechanism for bacteria from the central nervous system. ${ }^{29}$

\section{THE HOST'S RESPONSE}

Within 8-12 hr after inoculation of the pneumococcus, white blood cells (WBC) of the polymorphonuclear type appear in the CSF. Just preceding this is an opening of the blood-brain barrier extensively characterized by Scheld as an opening of the tight junctions between brain capillary endothelial cells associated with enhanced pinocytotic activity. ${ }^{26}$ This results in the influx of serum components, including chemotactic components of the complement system, especially C5a, which is in part responsible for the polymorphonuclear leukocyte (PMN) migration. ${ }^{8}$ It is significant that the appearance of WBC has no effect on the rate of proliferation of the bacteria, and neutropenic animals showed the same bacterial growth characteristics as normal animals. ${ }^{7}$ This lack of effect of granulocytes in the CSF against the encapsulated pathogen is a reflection of the low concentrations of opsonins (anti-capsular antibodies, complement)

\footnotetext{
${ }^{1}$ Department of Medicine, School of Medicine, University of Utah, Salt Lake City, UT 84132 and the ${ }^{2}$ Institute for Medical Microbiology,
} University of Berne, CH-3010 Berne, Switzerland. 
in this compartment of relative host defense deficiency. ${ }^{24,33}$ Consequently, when serum or anticapsular antibody was injected directly into the CSF prior to inoculation of the $S$. pneumoniae, the WBCs effectively killed the bacteria (W.M. Scheld, B. Perkins, and M.A. Sande, unpublished observations). Conversely, complement depletion of animals increases the virulence of pneumococci during experimental meningitis. ${ }^{42}$ One apparently effective host response in the CSF is the generation of fever in the rabbit. Bacterial growth is significantly reduced and the $\mathrm{ID}_{50}$ significantly increased in animals that were allowed to developed high temperatures compared to those in which the febrile response was suppressed. ${ }^{27,32}$

Another consistent pathophysiological alteration that developed during the first phase of infection in parallel with the opening of the blood-brain barrier was the decline of CSF glucose concentration (hypoglycorrhachia) and the increase in lactic acid concentration. ${ }^{4,13}$ Both changes were felt to represent increased utilization of glucose in response to relative cerebral ischemia caused by a reduction or mismatch of cerebral blood flow and a subsequent switch to the glycolytic cycle and away from the Krebs cycle of metabolism. ${ }^{10}$ This metabolic switch utilizes more glucose and produces lactate. It has been shown that tumor-necrosis factor- $\alpha$ (TNF- $\alpha$ ), when injected directly into the CSF, can induce this switch to anaerobic glycolysis with a subsequent increase in CSF lactate concentrations. ${ }^{45}$ Other studies have implicated reduced glucose transport as another potential cause of a low CSF glucose concentration. ${ }^{2}$

\section{MOLECULAR MEDIATORS OF INFLAMMATION IN MENINGITIS}

One of the most fruitful areas of research in bacterial meningitis was the exploration of the molecular mediators of inflammation. The question, how the host recognizes the presence of the pneumococcus in the subarachnoid space, and how it responds to this invasion could be addressed almost ideally in the Dacey/Sande rabbit model, in which substances can be directly injected into the CSF space and CSF can then be sampled almost continuously to monitor the response of the host. Work by several groups, notably Alex Tomasz and Elaine Tuomanen and their colleagues at Rockefeller University, has documented that fragments of the bacterial cell, not the bacterial polysaccharide capsule, are critical stimuli for the host's own inflammatory response. ${ }^{40,41}$ The pneumococcal cell wall is composed of a sugar backbone consisting of alternating molecules of $\mathrm{N}$-acetyl-muramic acid and $\mathrm{N}$-acetyl-glucosamine, which are connected to a three-dimensional network by pentapeptide side chains. In addition, the cell wall contains teichoic acid and lipoteichoic acids. All of these components of the cell wall are released as fragments from dying organisms undergoing autolysis and are able to induce mononuclear macrophages to express and release proinflammatory cytokines, such as TNF- $\alpha$, interleukin-1 (IL-1), IL-6, and many others. ${ }^{43}$ Release of cell wall fragments is dramatically increased immediately after initiation of antibiotic therapy, when large numbers of bacteria are killed, and the increased liberation of cell wall fragments leads to a heightened inflammatory response of the host. ${ }^{37,44}$ The exact potency of various cell wall fragments of the pneumococcus in inducing cytokines seems to vary, but small muramic acid dipeptides are thought to represent the minimal proinflammatory unit. The composition and amount of cell wall fragments released spontaneously or after initiation of antibiotic therapy can vary substantially from one pneumococcal strain to another. This variability may be one factor explaining the dramatic differences in the evolution of pathophysiologic changes, when different pneumococcal strains are injected into the CSF of rabbits. ${ }^{38}$

Induction of proinflammatory cytokines (TNF- $\alpha$, IL-1, IL-6) triggers a complex network of additional inflammatory mediators that, in concert, regulate the humoral and cellular inflammation during meningitis. Other proinflammatory cytokines, anti-inflammatory cytokines such as IL-10 and transforming growth factor- $\beta$ (TGF- $\beta$ ), soluble cytokine receptors, and receptor antagonists, several members of the more recently discovered class of chemokines, and lipid mediators such as platelet activating factor (PAF) all appear to be involved in the meningeal inflammation and the subsequent pathophysiologic changes (for a recent review, see ref. 35). The complexity of the inflammatory mediator network, and limitations of the currently used experimental systems rarely allow a conclusive determination as to what extent bacterial components or hostderived mediators are responsible for the changes observed during bacterial meningitis. It is possible that the only role of bacterial products is to start and maintain the inflammatory response of the host. Accordingly, inhibition of inflammatory mediators by administration of corticosteroids or nonsteroidal antiinflammatory drugs is effective in preventing the pathophysiologic changes in models of meningitis, particularly when the substances are given prior to the development of meningitis. ${ }^{36,44,47}$ Corticosteroids, with their broad mode of action, which includes potent inhibition of cytokines, seem more effective in downmodulating inflammation and pathophysiologic changes in meningitis than nonsteroidal antiinflammatory drugs. The latter class of drugs, represented for example by indomethacin, primarily influences the generation of some lipid mediators and has shown limited effects on the pathophysiology of meningitis. ${ }^{47}$

\section{ADVANCED PATHOPHYSIOLOGY OF MENINGITIS}

As a consequence of the increasing inflammatory reaction in the subarachnoid and ventricular space, meningitis leads to multiple, well-defined alterations of the intracranial physiology. These include brain edema, increased intracranial pressure, reduction in cerebral blood flow, and increased resistance to CSF resorption. ${ }^{30,36,46,48}$ Increased intracranial pressure represents probably the most critical single alteration that is both the result of other changes and contributes critically to cerebral ischemia with its devastating effect on the brain. ${ }^{48}$ Three factors can contribute to increased intracranial pressure during meningitis: brain edema, increased cerebral blood volume, and alterations of CSF hydrodynamics, in particular disturbed CSF resorption. ${ }^{30,31,49}$ Brain edema in meningitis appears to be a combination of vasogenic edema, resulting from the disruption of the blood-brain barrier, cytotoxic edema, resulting from cytotoxic mediators such as excitatory amino acids (EAA, see below), and interstitial edema. ${ }^{34}$ The latter is the consequence of 
a mechanical plugging of the CSF clearance system across the arachnoid villi in the superior saggital sinus by the inflammation in the subarachnoid space. ${ }^{30}$ White blood cells, fibrin, and bacteria collect in the villi, thus obstructing the CSF outflow and leading to increased CSF pressure, increased intracranial pressure, and possibly hydrocephalus. Downmodulation of inflammation with corticosteroids improves, as one would expect, the function of the CSF clearance system across the arachnoid villi. ${ }^{30}$ Finally, intracranial blood volume can also contribute to increased intracranial pressure. Early in the disease, increased blood flow to the brain (hyperemia) may be the most important contributing factor to increased intracranial blood volume and increased intracranial pressure. ${ }^{23}$ As the disease progresses, blood flow is reduced overall, but the venous blood volume may nevertheless be increased, leading to an increase of total intracerebral blood volume. ${ }^{49}$

The most significant consequence of increased intracranial pressure is its effect on cerebral blood flow. At least in severe cases of meningitis, typically caused by pneumococci, cerebral blood flow autoregulation is impaired and cerebral blood flow is directly dependent on cerebral perfusion pressure, which results from arterial pressure minus intracranial pressure. ${ }^{46,48}$ Thus, high intracranial pressure directly reduces cerebral perfusion pressure (particularly when the patient at the same time has a low systemic blood pressure because of dehydration or sepsis) and thus leads to reduced cerebral blood flow and subsequent cerebral ischemia. ${ }^{48,39}$ In practical terms, correction of cerebral perfusion pressure by normalizing arterial blood pressure and reducing intracranial hypertension is one of the most critical goals of the supportive management of patients with severe meningitis.

\section{MECHANISMS OF BRAIN DAMAGE}

The net result of the many changes occurring in the brain during meningitis is the development of brain damage, characterized by neuronal dropout and other, less well-defined cellular changes in the brain. As a clinical result, patients who survive the disease with neuronal damage show neurologic sequelae, such as learning deficits, mental retardation, sensorymotor deficits, and seizure disorders. ${ }^{9}$ The most common neurologic sequelae is hearing loss, which appears to result from inflammation affecting the inner ear after direct extension from the subarachnoid space along the cochlear aqueduct into the perilymphatic space of the cochlea. ${ }^{1,6}$ Molecular mediators of the processes that lead to the destruction of the hair cells of the inner ear are incompletely characterized, but may involve cytokines, oxygen-derived radicals, and, in the case of the pneumococcus, the bacteria-derived, highly potent cytotoxin pneumolysin. ${ }^{3}$

The molecular mechanisms that lead to neuronal damage during meningitis have started more recently to emerge from work in infant rats with experimental meningitis. Initial studies in this model were performed using group B streptococci as the infecting organism, but more recent experiments have shown that the pneumococcus produces very similar alterations as the group B streptococcus. The advantage of this model is the fact that substantial neuronal injury occurs as a result of the disease. ${ }^{15}$ This is in contrast to most other models of meningitis previously employed, particularly the rabbit model, where very little neuropathologic changes can be identified even in advanced meningitis. The new model has thus opened the door to investigate directly the role of various mediators in causing neuronal injury. The most important form of neuronal injury in this model involves focal cortical injury that resembles very much the focal damage seen in neonates and young children suffering from meningitis. ${ }^{15}$ The morphology of these changes shows all of the features of ischemic cortical damage, i.e., it is wedgeshaped and most severe in watershed areas of the cerebral circulation. Indeed, bloodflow studies have documented severe focal ischemia in the model in a pattern that was identical to the histopathologic changes observed. Further supporting the ischemic nature of the cortical injury were studies that showed improved neurologic outcome in animals, in which the extent of blood flow changes was reduced, and worsened neurologic outcome, when blood flow changes were aggravated. Thus, molecular mediators of blood flow alterations during meningitis became an important area of investigation in this model.

Early in experimental meningitis, cerebral blood flow increases as a result of vasodilation. Work in an adult rat model of pneumococcal meningitis by Walter Pfister's group in Munich has documented that this early vasodilation is mediated by nitric oxide (NO). ${ }^{16}$ We have found subsequently that NO continues to play an important role as regulator of cerebral blood flow far into the course of the disease. ${ }^{19}$ We observed that inhibition of the inducible nitric oxide synthase (NOS), which is upregulated during meningitis in the subarachnoid space inflammation and vasculature, and which is responsible at least for part of the NO produced during meningitis, led to a harmful increase in cerebral ischemia in animals with advanced meningitis. This increased ischemia was associated with an increase in neuronal damage. Thus, NO produced by the inducible NOS in or close to the cerebral vasculature has a beneficial effect, because its vasodilative effect counteracts other processes that tend to lead to vasoconstriction and subsequent ischemia.

Some of the vasoconstrictive mediators have also been identified. Most notable are oxygen-derived radicals, such as superoxide, hydrogen peroxide, and others. These metabolic products of essential biologic processes, such as mitochondrial respiratory chain and activation of macrophages, have multiple harmful effects on cells and macromolecules, including lipid peroxidation, DNA damage, and protein oxidation. In the infant rat model of meningitis, lipid peroxidation is strongly increased in advanced disease, and histochemical methods have allowed direct localization of the production of superoxide to the subarachnoid space and the cerebral vasculature. ${ }^{17}$ Importantly, scavenging of these radicals by so-called spin-trapping agents, which can bind and detoxify radicals, led to a reduction of lipid peroxidation, but also to an improvement of cerebral blood flow with associated reduction of ischemic neuronal damage. ${ }^{17}$ Thus, oxidative radicals (by mechanisms that are currently not completely understood) are important mediators of vasoconstriction during meningitis, and their inhibition is beneficial by improving cerebral blood flow. Another molecule with vasoconstrictive properties that has recently been implicated in the ischemia developing during meningitis is endothelin. This vasoactive peptide is increased in the CSF of patients with meningitis, and we found that an endothelin-receptor antagonist, bosentan, dramatically improved the neurologic out- 
come of meningitis in the infant rat model, apparently at least in part by improving cerebral blood flow (Pfister et al., in press). Other mediators of vasoconstriction likely play also a role in meningitis and will need to be identified in future studies. It is likely that increased local coagulation leads to thrombosis with associated disturbances of cerebral blood flow, but this has not been investigated in any of the available models of meningitis.

Some of the molecules that are directly neurotoxic during meningitis have also been identified. Because ischemia appears to play a critical role in causing brain injury, studies have focused on the role of excitatory amino acids (EAA) in causing neuronal cell death. EAA (e.g., glutamate) are physiologic neurotransmitters that are released at increased concentrations from neurons subjected to stress, such as ischemia or hypoglycemia. Their increased concentrations in the extracellular fluid leads to overstimulation of postsynaptic EAA receptors, in particular the NMDA receptor, with subsequent increased influx of calcium through the receptor channel, activation of various cellular pathways, including production of NO by neuronal NOS, and generation of oxidative radicals. All of these processes ultimately lead to cellular death. This appears to be an important mechanism of cell death in stroke. In models of meningitis, we and others have documented an increase in glutamate concentrations both in the CSF and, more importantly, in the intercellular fluid, and have shown that an antagonist of the NMDA receptor, kynurenic acids, is neuroprotective. ${ }^{11,18,22}$ Thus, EAA represent the first neurotoxic molecule shown directly to play a role in bacterial meningitis.

One consequence of overstimulation of NMDA receptors is the generation of NO by neurons containing neuronal NOS. NO can potentially be neurotoxic directly, as shown in brain cell culture systems stimulated with pneumococcal cell walls, ${ }^{14}$ or it can combine with superoxide to form the highly cytotoxic molecule peroxynitrite (ONOO). Superoxide is likely to be formed during meningitis in the brain, either as a result of ischemic insult and the associated activation of the glycolytic pathway, or as a result of activation of microglia and other phagocytic cells by cytokines and bacterial products. Preliminary evidence suggests that peroxynitrite is indeed formed in the brain during experimental meningitis, and that it contributes to the development of neuronal injury. In the infant rat model, we found evidence for increased production of nitrotyrosine, the chemical hallmark of the presence of peroxynitrite, by immunocytochemistry and high-performance liquid chromatography (HPLC) (unpublished observations). A role of peroxynitrite in meningitis was further supported by treatment studies. Both a scavenger of peroxynitrite (uric acid), and an inhibitor (3-aminobenzamine) of the enzyme poly-(ADP-ribose) polymerase (PARP), which is activated by the action of peroxynitrite on mitochondria, significantly reduced neuronal injury in the treated compared to the untreated animals. ${ }^{12}$

Thus, molecules that contribute to the demise of neurons during meningitis may emerge as targets for adjunctive therapy. It would appear that a combined inhibition of oxidative radicals, EAA, and peroxynitrite should have profound effects on the development of neuronal injury. Such therapies could be effective even when instituted in relatively advanced stages of the disease, since the interrupted pathways are late events in the sequence leading from subarachnoid space inflammation to neuronal injury. Specific neuroprotective therapies could represent a potential advantage over the use of corticosteroids, which act primarily by downmodulating inflammation and thus at a relatively early stage in the pathophysiologic cascade of meningitis. This effect primarily on early events in meningitis may, at least in part, be a reason why corticosteroids, although clearly beneficial in several clinical studies, have shown overall modest benefits. This has led to a continued controversy regarding their usefulness in meningitis. ${ }^{20,25}$ We hope that the lessons that have been and will be learned from the use of models such as the Dacey/Sande rabbit model will lead to new therapies that can improve the outcome of bacterial meningitis.

\section{REFERENCES}

1. Bhatt, S.M., A. Lauretano, C. Cabellos, C. Halpin, R.A. Levine, W.Z. Xu, J.B.J. Nadol, and E. Tuomanen. 1993. Progression of hearing loss in experimental pneumococcal meningitis: correlation with cerebrospinal fluid cytochemistry. J. Infect. Dis. 167:675-683.

2. Brooke-Williams, R. 1964. Alterations in the glucose transport mechanism in patients with complications of bacterial meningitis. Pediatrics 34:491-502.

3. Comis, S.D., M.P. Osborne, J. Stephen, M.J. Tarlow, T.L. Hayward, T.J. Mitchell, P.W. Andrew, and G.J. Boulnois. 1993. Cytotoxic effect on hair cells of guinea pig cochlea produced by pneumolysin, the thiol activated toxin of Streptococcus pneumoniae. Acta Otolaryngol. 113:152-159.

4. Cooper, A., H. Beaty, S. Oppenheimer, R. Goodner, and R. Petersdorf. 1968. Studies on the pathogenesis of meningitis. Glucose transport and spinal fluid production in experimental pneumococcal meningitis. J. Lab. Clin. Med. 71:473-483.

5. Dacey, R.G., and M.A. Sande. 1974. Effect of probenecid on cererbrospinal fluid concentrations of penicillin and cephalosporin derivatives. Antimicrob. Agents Chemother. 6:437-441.

6. Dodge, P.R., D. Hallowell, R.D. Feigin, S.J. Holmes, S.L. Kaplan, D.P. Jubelirer, B. Stechberg, and S.K. Hirsh. 1984. Prospective evaluation of hearing impairment as a sequela of acute bacterial meningitis. N. Engl. J. Med. 311:869-874.

7. Ernst, J.D., J.M. Decazes, and M.A. Sande. 1983. Experimental pneumococcal meningitis: role of leukocytes in pathogenesis. Infect. Immun. 41:275-279.

8. Ernst, J.D., K. Hartiala, I.M. Goldstein, and M.A. Sande. 1984. Complement (C5)-derived chemotactic activity accounts for accumulation of polymorphonuclear leukocytes in cerebrospinal fluid of rabbits with pneumococcal meningitis. Infect. Immun. 46:81-86.

9. Grimwood, K., V.A. Anderson, L. Bond, C. Catroppa, R.L. Hore, E.H. Keir, T. Nolan, and D.M. Robertson. 1995. Adverse outcome of bacterial meningitis in school-age survivors. Pediatrics 95:646-656.

10. Guerra-Romero, L., M.G. Täuber, M.A. Fournier, and J.H. Tureen. 1992. Lactate and glucose concentrations in brain interstitial fluid, cerebrospinal fluid, and serum during experimental pneumococcal meningitis. J. Infect. Dis. 166:546-550.

11. Guerra-Romero, L., J.H. Tureen, M.A. Fournier, V. Makrides, and M.G. Täuber. 1993. Amino acids in cerebrospinal and brain interstitial fluid during experimental pneumococcal meningitis. Pediatr. Res. 33:510-513.

12. Ho, T.C., L. Chow, D.M. Ferriero, M.G. Täuber, and J.H Tureen. 1998. Peroxynitrite-mediated brain injury in experimental group B streptococcal meningitis in the neonatal rat. J. Invest. Med. 46:155.

13. Hochwald, G., S. Nakamura, R. Chase, and J. Gorelick. 1984. Cerebrospinal fluid glucose and leukocyte responses in experimental meningitis. J. Neurol. Sci. 63:381-391.

14. Kim, Y.S., S. Kennedy, and M.G. Täuber. 1995. Toxicity of 
Streptococcus pneumoniae in neurons, astrocytes, and microglia in vitro. J. Infect. Dis. 171:1363-1369.

15. Kim, Y.S., R.A. Sheldon, B.R. Elliot, Q. Liu, D.M. Ferriero, and M.G. Täuber. 1995. Brain damage in neonatal meningitis caused by group B streptococci in rats. J. Neuropathol. Exp. Neurol. 54:531-539.

16. Koedel, U., A. Bernatowicz, R. Paul, K. Frei, A. Fontana, and H.W. Pfister. 1995. Experimental pneumococcal meningitis: cerebrovascular alterations, brain edema, and meningeal inflammation are linked to the production of nitric oxide. Ann. Neurol. 37:313-323.

17. Leib, S.L., Y.S. Kim, L.L. Chow, R.A. Sheldon, and M.G. Täuber. 1996. Reactive oxygen intermediates contribute to necrotic and apoptotic neuronal injury in an infant rat model of bacterial meningitis due to group B streptococci. J. Clin. Invest. 98:2632-2639.

18. Leib, S.L., S.Y. Kim, D.M. Ferriero, and M.G. Täuber. 1996 Neuroprotective effect of excitatory amino acid antagonist kynurenic acid in experimental bacterial meningitis. J. Infect. Dis. 173: $166-171$

19. Leib, S.L., Y.S. Kim, S.M. Black, J.H. Tureen, and M.G. Täuber. 1998. Inducible nitric oxide synthase and the effect of aminoguanidine in experimental neonatal meningitis. J. Infect. Dis. 177:692-700

20. McIntyre, P.B., C.S. Berkey, S.M. King, U.B. Schaad, T. Kilpi, G.Y. Kanra, and C.M. Odio Perez. 1997. Dexamethasone as adjunctive therapy in bacerial meningitis. A meta-analysis of randomized clinical trials since 1988. J. Am. Med. Assn. 278:925-931.

21. Moxon, E.R., and P.A. Murphy. 1978. Haemophilus influenzae bacteremia and meningitis resulting from the survival of a single organism. Proc. Natl. Acad. Sci. USA 75:1534-1536.

22. Perry, V., R.S.K. Young, W.J. Aquilla, and M.J. During. 1993. Effect of experimental Escherichia coli meningitis on concentrations of excitatory and inhibitory amino acids in the rabbit brain in vivo microdialysis study. Pediatr. Res. 34, 187-191.

23. Pfister, H.W., U. Koedel, R.L. Haberl, U. Dirnagl, W. Feiden, L.G. Kuckdesche, and K.M. Einhaupl. 1990. Microvascular changes during the early phase of experimental bacterial meningitis. J. Cereb. Blood Flow Metab. 10:914-922.

24. Propp, R.P., B. Jannari, and K. Barron. 1977. Measurement of the third component of complement in cerebrospinal fluid by modified electroimmunodiffusion. Scand. J. Clin. Lab. Invest. 37:385-390.

25. Quagliarello, V.J., and W.M. Scheld. 1997. Treatment of bacterial meningitis. N. Engl. J. Med. 336:708-716.

26. Quagliarello, V.J., W.J. Long, and W.M. Scheld. 1986. Morphologic alterations of the blood-brain barrier with experimental meningitis in the rat. J. Clin. Invest. 77:1085-1095.

27. Sande, M.A., E.R. Sande, J.D. Woolwine, C.J. Hackbarth, and P.M. Small. 1987. The influence of fever on the development of experimental Streptococcus pneumoniae meningitis. J. Infect. Dis. 156:849-850.

28. Scheld, W.M., and M.A. Sande. 1983. Bactericidal versus bacteriostatic antibiotic therapy of experimental pneumococcal meningitis in rabbits. J. Clin. Invest. 71:411-419.

29. Scheld, W.M., T. Park, R.G. Dacey, H.R. Winn, J.A. Jane, and M.A. Sande. 1979. Clearance of bacteria from cerebrospinal fluid to blood in experimental meningitis. Infect. Immun. 24:102-106.

30. Scheld, W.M., R.G. Dacey, H.R. Winn, J.E. Welsh, J.A. Jane, and M.A. Sande. 1980. Cerebrospinal fluid outflow resistance in rabbits with experimental meningitis. J. Clin. Invest. 66:243-253.

31. Schuchat, A., K. Robinson, J.D. Wenger, L.H. Harrison, M. Farley, A.L. Reingold, L. Lefkowitz, and B.A. Perkins. 1997. Bacterial meningitis in the United States in 1995. N. Engl. J. Med. 337:970-976.

32. Small, P.M., M.G. Täuber, C.J. Hackbarth, and M.A. Sande. 1986. Influence of body temperature on bacterial growth rates in experimental pneumococcal meningitis in rabbits. Infect. Immun. 52:484-487.
33. Smith, H., B. Bannister, and M.J. O'Shea. 1973. Cerebrospinal fluid immunoglobulins in meningitis. Lancet 1:591-593.

34. Täuber, M.G. 1989. Brain edema, intracranial pressure and cerebral blood flow in bacterial meningitis. Pediatr. Infect. Dis. J. 8:915-917.

35. Täuber, M.G., and B. Moser. 1999. Cytokines and chemokines in meningeal inflammation: Biology and clinical implications. Clin. Infect. Dis. 28:1-11.

36. Täuber, M.G., H. Khayam-Bashi, and M.A. Sande. 1985. Effects of ampicillin and corticosteroids on brain water content, CSF pressure and CSF lactate in experimental pneumococcal meningitis. J. Infect. Dis. 151:528-534.

37. Täuber, M.G., A.M. Shibl, C.J. Hackbarth, J.W. Larrick, and M.A. Sande. 1987. Antibiotic therapy, endotoxin concentration in cerebrospinal fluid, and brain edema in experimental Escherichia coli meningitis in rabbits. J. Infect. Dis. 156:456-462.

38. Täuber, M.G., M. Burroughs, U.M. Niemöller, H. Kuster, U. Borschberg, and E. Tuomanen. 1991. Differences of pathophysiology in experimental meningitis caused by three strains of Streptococcus pneumoniae. J. Infect. Dis. 163:806-811.

39. Täuber, M.G., E. Sande, M.A. Fournier, J.H. Tureen, and M.A. Sande. 1993. Fluid administration, brain edema, and cerebrospinal fluid lactate and glucose concentrations in experimental Escherichia coli meningitis. J. Infect. Dis. 168:473-476.

40. Tuomanen, E., H. Liu, B. Hengstler, O. Zak, and A. Tomasz. 1985. The induction of meningeal inflammation by components of the pneumococcal cell wall. J. Infect. Dis. 152:859-868.

41. Tuomanen, E., A. Tomasz, B. Hengstler, and O. Zak. 1985. The relative role of bacterial cell wall and capsule in the induction of inflammation in pneumococcal meningitis. J. Infect. Dis. 151:535-540.

42. Tuomanen, E., B. Hengstler, O. Zak, A. Tomasz. 1986. The role of complement in inflammation during experimental pneumococcal meningitis. Microb. Pathogen. 1:15-32.

43. Tuomanen, E., B. Hengstler, O. Zak, and A. Tomasz. 1986. Induction of meningeal inflammation by diverse bacterial cell walls. Eur. J. Clin. Microbiol. 5:682-684.

44. Tuomanen, E., B. Hengstler, R. Rich, M.A. Bray, O. Zak, and A. Tomasz. 1987. Nonsteroidal anti-inflammatory agents in the therapy for experimental pneumococcal meningitis. J. Infect. Dis. 155:985-990.

45. Tureen, J. 1995. Effect of recombinant human tumor necrosis factor-alpha on cerebral oxygen uptake, cerebrospinal fluid lactate, and cerebral blood flow in the rabbit: role of nitric oxide. J. Clin. Invest. 95:1086-1091.

46. Tureen, J.H., Dworkin, R.J., S.L. Kennedy, M. Sachdeva, and M.A. Sande. 1990. Loss of cerebrovascular autoregulation in experimental meningitis in rabbits. J. Clin. Invest. 85:577-581.

47. Tureen, J.H., Täuber, M.G., and M.A. Sande. 1991. Effect of indomethacin on the pathophysiology of experimental meningitis in rabbits. J. Infect. Dis. 163:647-649.

48. Tureen, J.H., M.G. Täuber, and M.A. Sande. 1992. Effect of hydration status on cerebral blood flow and cerebrospinal fluid lactic acidosis in rabbits with experimental meningitis. J. Clin. Invest. 89:947-953.

49. Tureen, J., Q. Liu, and L. Chow. 1996. Near-infrared spectroscopy in experimental pneumococcal meningitis in the rabbit: cerebral hemodymamics and metabolism. Pediatr. Res. 40:759-763.

Address reprint requests to: M. Täuber, M.D. Institute for Medical Microbiology Friedbühlstr. 51 CH-3010 Berne Switzerland E-mail: taeuber@imm.unibe.ch 